\section{Investigation on deformation behavior in the surface of metal foil with Ultrasonic Vibration-assisted micro- forging.}

\author{
Zidong Yin ${ }^{1, a}$, Ming Yang ${ }^{2, b}$. \\ ${ }^{1}$ Graduate School of System Design, Tokyo \\ Metropolitan University, 6-6, Asahigaoka, \\ Hino-shi 191-0065, Tokyo, Japan \\ ${ }^{2}$ Graduate School of System Design, Tokyo \\ Metropolitan University, 6-6, Asahigaoka, \\ Hino-shi 191-0065, Tokyo, Japan \\ ayin-zidong@ed.tmu.ac.jp, b yang@tmu.ac.jp
}

Keywords: surface finishing; surface deformation; micro-forging; ultrasonic vibration-assisted.

\begin{abstract}
Excitation of the acoustic field leading to the Blaha effect affects the plasticity of the material significantly in ultrasonic vibration-assisted forming. In a microforming field, the effects are more significant in the deformation in surface of materials [1]-[3], in which reduction of the surface roughness based on the increasing of plastic deformation of surface asperity was effective ${ }^{[4]}$. On the other hand, the effect on deformation behavior of the bulk region indicted reduction in the yield stress of materials, and not only acoustic effect ${ }^{[5]}$, but also impact effect is found to generate a large amount of dislocation and produce plastic deformation ${ }^{[6][7]}$. However, the effect on the bulk is more significant as that on the surface. Differences in the effect on the surface and the bulk are not clarified. In this study, the mechanism of the deformation in the surface of the material with ultrasonic vibration assistance is investigated and compared with that in the bulk. Forging tests using a newly developed ultrasonic vibrator were carried out on pure $\mathrm{Cu}$ foils with various process conditions. The longitudinal vibration frequency of the ultrasonic transducer is $60 \mp 2 \mathrm{kHz}$, and the vibration amplitude is in an adjustable range of $0 \sim 10$

$\mu \mathrm{m}$. Forging test was carried out at different initial stress, specimen size and amplitude. The difference in acoustic softening and impact effects on the surface and the bulk was discussed.
\end{abstract}

\section{Introduction}

Due to the increasing demand for micro technical products, such as micro-electro-mechanical systems (MEMS), the process of micro forming has been widely used to produce miniature metallic parts. Nevertheless, some problems so-called size effect will be caused by scaling-down like the ratio of roughness to its dimension decreasing, increased forming stress, and lower forming accuracy in the process behavior ${ }^{[8]}$. In the decade, plenty of researchers have tried to use different extra energies to increase surface properties and form accuracy. Compared with others, Ultrasonic vibration assistance, as a relatively higher efficient and lower energy losing processing method in the field of micro forming, has garnered more attention from both academic and industrial fields.

From 1955, Austrian scientists Blaha and Langenecker found that ultrasonic vibration could reduce the yield stress significantly in the tensile test of Zinc single crystal for the first time ${ }^{[9]}$. Researchers have made much effort to apply Ultrasonic vibration into micro-manufacturing devices. Jimma et al. (1998) designed an experimental apparatus applying ultrasonic vibration to a deep drawing process, which increased the limiting drawing ratio in both rolled steel and 304 stainless steel ${ }^{[10]}$. Weidong Zhai and li Yanle(2019) study the ultrasonic-assisted incremental sheet forming process, and the result shows that forming force is considerably reduced, and bearing capacity can be improved. Both of these effects are more obvious at step-down size or the thin material sheet ${ }^{[11]}$. Chunju Wang(2017) presented copper foil 's mechanical properties with ultrasonic vibration tensile testing especially considering the size effect, and the result shows the flow stress is decreased by ultrasonic vibration by different geometry sizes and more significant when the size was scaling down ${ }^{[12]}$.

Previous research has established that ultrasonic affects materials in two aspects, volume and surface. The effect on the volume was reported that when ultrasonic vibration is applied to the tool, reduction of the yield stress of materials will happen to a certain degree. As for effect on the surface, Bai and Yang (2016) got better surface finishing by an innovative method applied ultrasonic vibration, surface asperities deformation and reduction of surface roughness was linear to ultrasonic vibration amplitude and static stress has been provided ${ }^{[13]}$. But the fundamental mechanisms of ultrasonic affecting material is still controversial. There are two explanations accepted generally on a macro scale, stress superposition and acoustic softening. Stress superposition is only an apparently average stress reduction with periodically loading and unloading, which is explained as the framework of elastic-plastic behavior of the material under uniaxial deformation (Kirchner et al., 1985) and it is thought that no microstructural changes are induced by stress superposition $^{[14]}$. On the other hand, acoustic softening works by decreasing the real flowing stress and changing the material properties or microstructure (Langenecker. 1966) ${ }^{[15]}$. Ultrasonic offers energy for dislocation, which can be part of required activation energy for dislocations to overcome lattice obstacles so that the mobility of dislocation is enhanced. J. Hu, et al (2020) showed that when acoustic softening happens, compared to the condition without ultrasonic vibration, the dislocations/low-angle grain boundaries distribute randomly in grains, which results motions of 


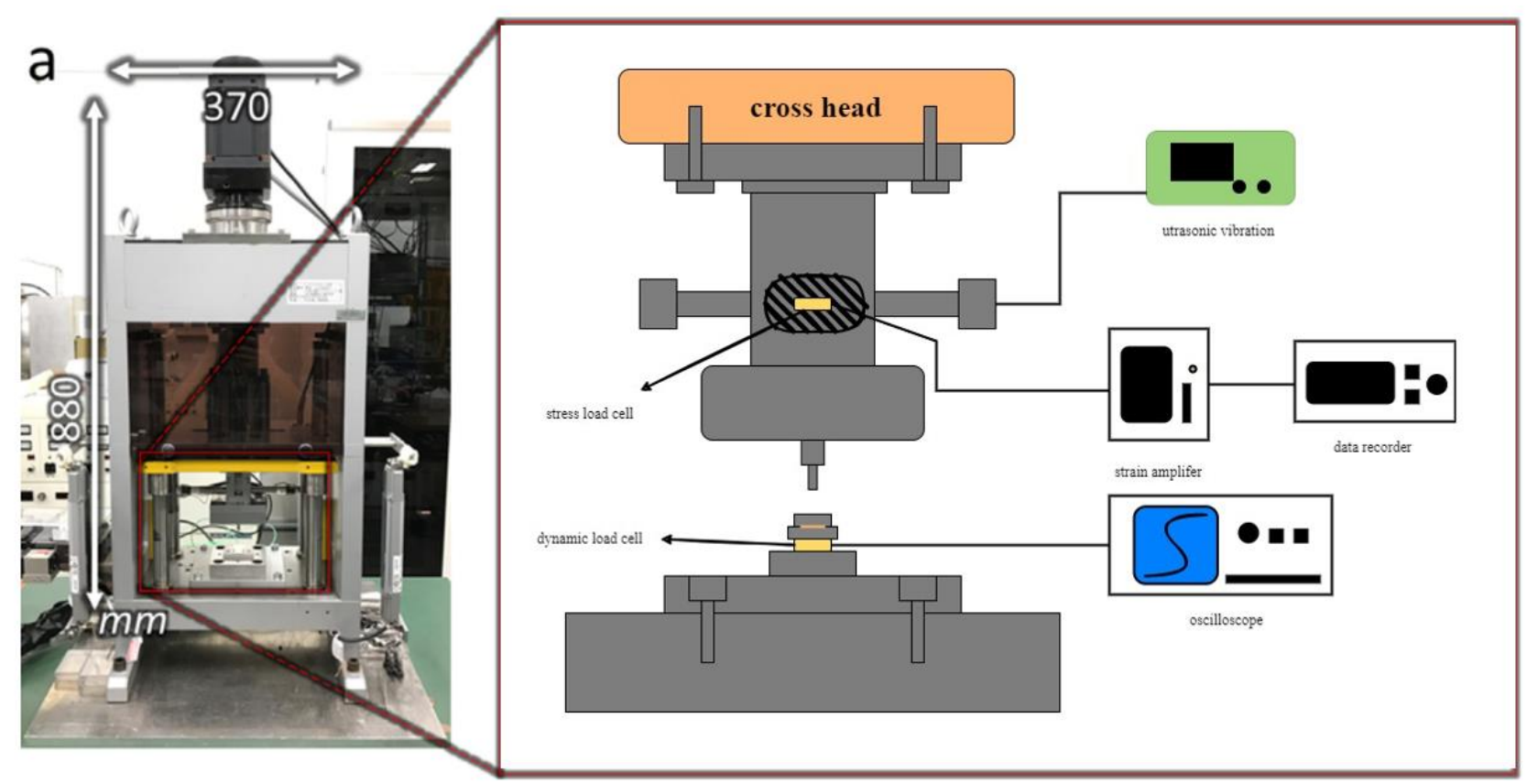

Fig. 1. Configuration of the ultrasonic-assisted micro-forging system, (a) Appearance of desktop miniature servo press machine and (b) Schematic illustration of the micro-froging system.

dislocations/low-angle grain boundaries is improved by acoustic softening ${ }^{[5]}$. At the micro-level, it is expressed as stress reduction. Furthermore, excepting stress superposition and acoustic softening, dynamic impact is reported as an important role in an ultrasonic assistant compression test. In the ultrasonic vibration assistant compression test, the dynamic effect happens whether the vibrated punch is able to detach from the specimen or not periodically. The deformation caused by the ultrasonic dynamic impact effect is accumulated by the repeated impact from punch and bottom die gradually. The grain size is refined and low-angle grain boundaries appear in the top side and center of the material.

Indeed, Some researchers have been studies how ultrasonic affects the surface of the material. It is demonstrated that at the same vibration time, the forming work is increasing with frequency, better surface finishing and higher microhardness could be gained with higher frequency (Yang Bai 2013) ${ }^{[3]}$. The impact effect will gradually become more dominating when the amplitude of ultrasonic vibration increases, which would lead to more reduction of forming force and more change of microstructure (J. Hu et al) ${ }^{[7]}$. Whereas, no study focused on how the impact effect from ultrasonic vibration works on the surface of the material, like reducing the surface roughness. Not to mention comparing the differences induced by ultrasonic in the surface and inner material. In order to clarify the mechanism of the deformation in the surface of the material, it is worth studying the acoustic effect and impact effect respectively.

In this study, to investigate the deformation behavior in the surface under ultrasonic vibration in microscale, considering the dynamic impact effect and acoustic effect, a novel ultrasonic-assisted micro-forging will be used. Micro-forging tests of commercial copper were carried out in different conditions. Effects of amplitudes on surface topographies, reduction of surface roughness, and deformation of surface asperities will be discussed. Additionally, in order to investigate the difference of micro-structure between the top side and deep side of material during micro-forging under diverse amplitude, EBSD ( electron backscatter diffraction) will be used for a better understanding of the underlying mechanisms.

\section{Experimental setup}

Ultrasonic-assisted micro-forging test system. in this study, a novel ultrasonic-assisted micro-forging test system including an upper die assembly, a lower die, and an ultrasonic vibrator is set up, as shown in Fig. 1. The ultrasonic vibrator contains two horizontal ultrasonic transducers, a horn at a resonance frequency of $60 \mathrm{kHz}$ and a punch for forging.

As shown in Fig.2, it is the integrated structure of the ultrasonic vibrator with the aid of FE modal analysis.

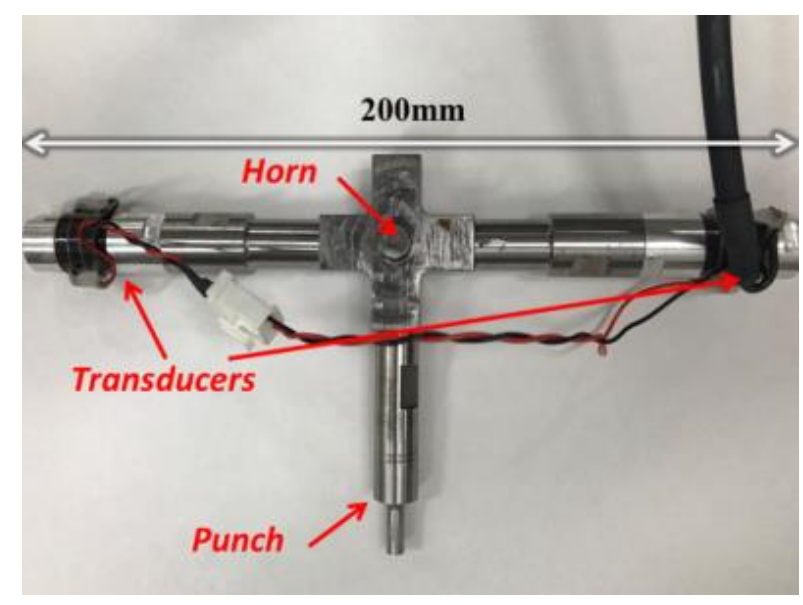

Fig. 2. Integrated structure of ultrasonic bibrator 
Two horizontal transducers generated ultrasonic vibration originally in the same phase. Then the punch receives the vibration, which is amplified and transformed to a vertical direction by a specially designed horn. Finally, the maximum amplitude of $6 \mu \mathrm{m}$ on the punch tip is measured by laser displacement meter (LC-2400). The input electrical signal for ultrasonic transducers is a sine wave with a frequency of $60 \mathrm{kHz}$ from an ultrasonic signal generator. The die and punch are made from tool steel (SKD11), and the surfaces are fine grinded with the heat treatment, keeping the surface roughness on $\mathrm{Ra}=0.1 \mu \mathrm{m}$. Punch surface will be re-ground to keep surface clearing and ensure no specimen residual after every experiment. And a stress load cell located on the ultrasonic vibrator to measure the pre-load, and since it connects to the strain amplifier, the strain can also be obtained. In order to prevent the warping of the copper chip during processing, a plate with four screws distributed in four corners and a through-hole for punch in the center is processed, as shown in Fig. 3, which can ensure the specimen is fixed tightly.

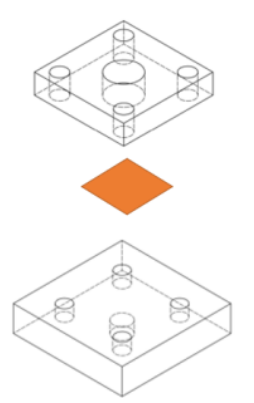

(a)

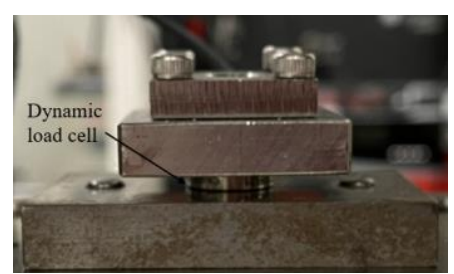

Fig. 3. The fixation system of specimens.(a) Schematic illustration of fixation system(b)photo of fixation system

Additionally, a dynamic load cell (Kistler 9132B), oscilloscope(Tektronix, DPO2014), and data recorder (OMRON ZR-RX70) are made up of a data collection module to measure the dynamic force. Because of the high rigidity of dynamic load cell (Kistler 9132B) is suitable for measuring rapidly changing force. Its reliability could be proved by the waveforms from the oscilloscope at $60 \mathrm{kHz}$ in the latter experiment, As shown in Fig.4.

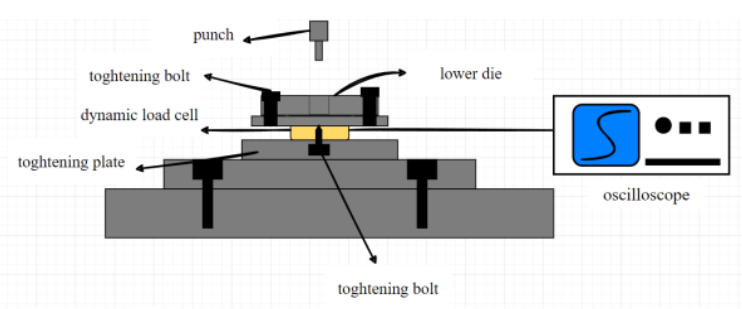

Fig. 4. Dynamic force test system

\section{Specimen and forging test procedure.}

Commercially copper was selected to make forging test samples, which were machined to a thin chip which length, width, and height in size $10 \mathrm{~mm}, 10 \mathrm{~mm}$, and
$0.2 \mathrm{~mm}$. Since all the small pieces of chips are cut from a big one, the initial surface roughness can be in $\mathrm{Rs}=220 \mathrm{~nm}$. The chemical composition of specimen is shown in Table 1.

For getting the impact effect under relatively low amplitude, the initial pre-load is set to $100 \mathrm{~N}$. since higher pre-load would make the punch departing from surface of material become more harder (Bai Yang 2014) [4], which means the impact effect would be observed more difficult. Vibration would keep 10s in every processing, because at same frequency, the plastic deformation only happens in the initial $2 \mathrm{~s}$, after this, the deformation is very limit (Yang Bai, 2014) ${ }^{[1]}$.

Consequently, the forging test procedure, shown like Fig. 4, were carried out at and ten specimens were relatively processed by 10 different amplitudes of $0.5,1$, $1.5,2,2.5,3,3.5,4,5,6 \mu \mathrm{m}$. The frequency in this study is always $60 \mathrm{Khz}$.

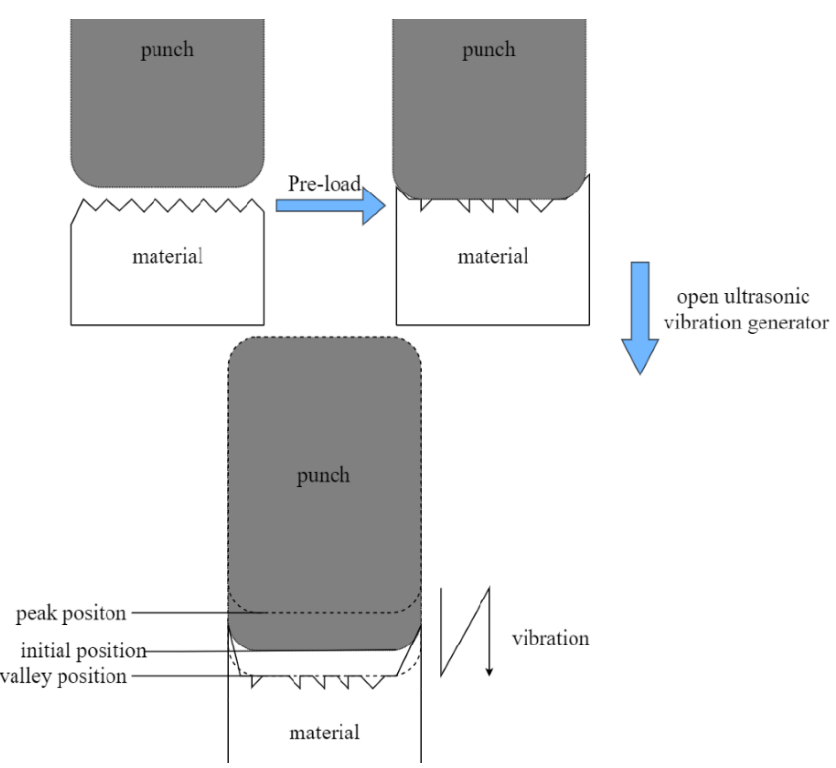

Fig. 5. Procedure of mic-forging of the punch

\section{Results and discussion}

Separation of acoustic effect and impact effect

According to the explanation of impact effect, the judgment standard of occurrence of impact effect is whether punch can contact and detach from the surface of specimen periodically during the process (Bai and Yang, 2014) ${ }^{[3]}$. In the case of the forging test, when a pre-load is loaded, a small amount of elastic deformation would be created on the surface of the copper chip. At that moment, the punch stays at an initial position. Then the vibration starts, the punch would motivate between peak position and valley position, as shown in Fig. 5. At the low amplitude, if the created deformation by the punch stayed at valley position is not enough to make plastic deformation happen, the elastic spring back would make the material of surface recover to the original state following the punch when it moves from valley position to peak position and finish a period of vibration. 


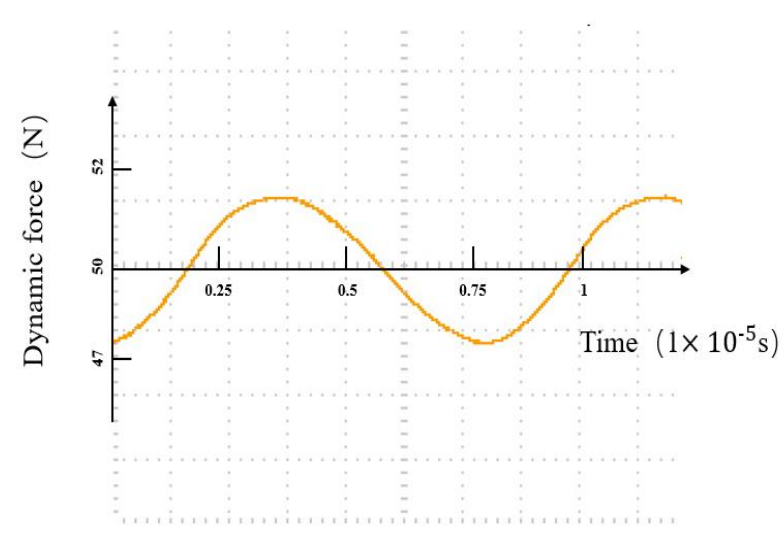

(a) $1 \mu \mathrm{m}$

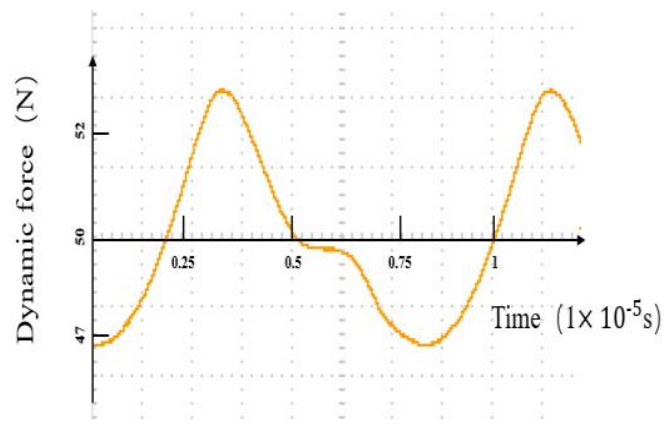

(c) $3 \mu \mathrm{m}$

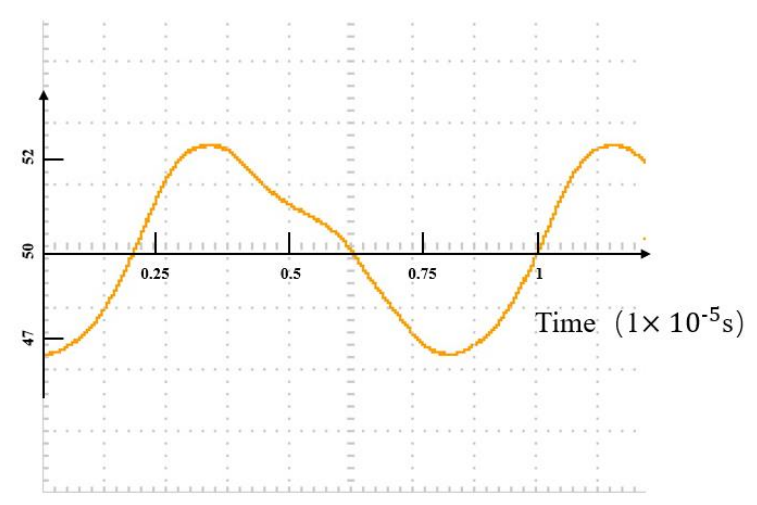

(b) $2 \mu \mathrm{m}$

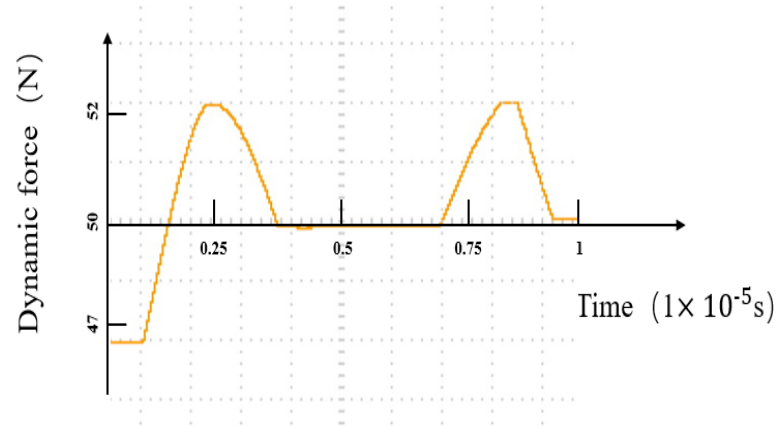

(d) $6 \mu \mathrm{m}$

Fig. 6. waveform of dynamic oscillatory forces during compression with different ultrasonic amplitudes.

As the result, the punch would contact the surface of the specimen during the whole process, which means the impact effect couldn't happen in this situation. Besides, the elastic force would be larger when the punch stays at valley position and reduce gradually when the punch move from valley position to peak position, so the dynamic force would be captured by the dynamic load cell and converted to input electrical signal, then displayed as an ideal sine wave on an oscilloscope. Fig .6(a) shows the waveform on the oscilloscope under $1 \mu \mathrm{m}$ amplitude.

To the contrary situation, with the amplitude increasing, there is an appearance of distortion on the waveform, shown as Fig .6(b). it is mainly due to when the amplitude keeps increasing, and it makes the displacement of the punch deeper. Some parts of the asperity on the surface, especially the bigger size, start plastic deformation, leading to the elastic force sudden reduction while the punch is moving back to the peak position. And a tiny slope appears on the waveform. Furthermore, if the amplitude continues increasing, the slope of the distortion part on the waveform tends to flatten, shown as Fig.6(c), which indicates more plastic deformation happened on surface asperity. When the amplitude reaches to $6 \mu \mathrm{m}$, which is the maximum of the ultrasonic vibration, a straight line appears. This is to say, a majority of asperity on the surface have started plastic deformation. Only a few still have spring-back. The dynamic load cell can hardly detect the elastic force.

According to the waveform displayed on the oscilloscope, the acoustic effect and impact effect could be separated successfully in this study. At the amplitude of $0.5,1,1.5 \mu \mathrm{m}$, only acoustic effects work in the process. After the amplitude over $1.5 \mu \mathrm{m}$, the impact effect would appear in the process. And with increasing amplitude, the impact effect is more significant.

\section{Surface finishing by acoustic effect and impact} effect.

To investigate which acoustic and dynamic effects are more significant in the micro-forging test, the surface topographies of copper chips are detected by an atomic force microscope (Keyence VN-8010). The surface topographies of specimens of the original surface and forging under different amplitude of $0.5,1$, 1.5, 2, 3.5, 6 $\mu \mathrm{m}$ are shown in Fig. 7(a)-(g), respectively.

Original surface roughness (Rs) of $220 \mathrm{~nm}$ was obtained before forging test, and the maximum height of asperity reached about $5630.1 \mathrm{~nm}$, as shown in Fig. 7(a). after being processed by amplitude $0.5 \mu \mathrm{m}$, the highest asperity decreased to $2419 \mathrm{~nm}$, however, with the amplitude increasing to 1 or $1.5 \mu \mathrm{m}$, which means the impact effect has not started yet, there is no noticeable reduction on the 

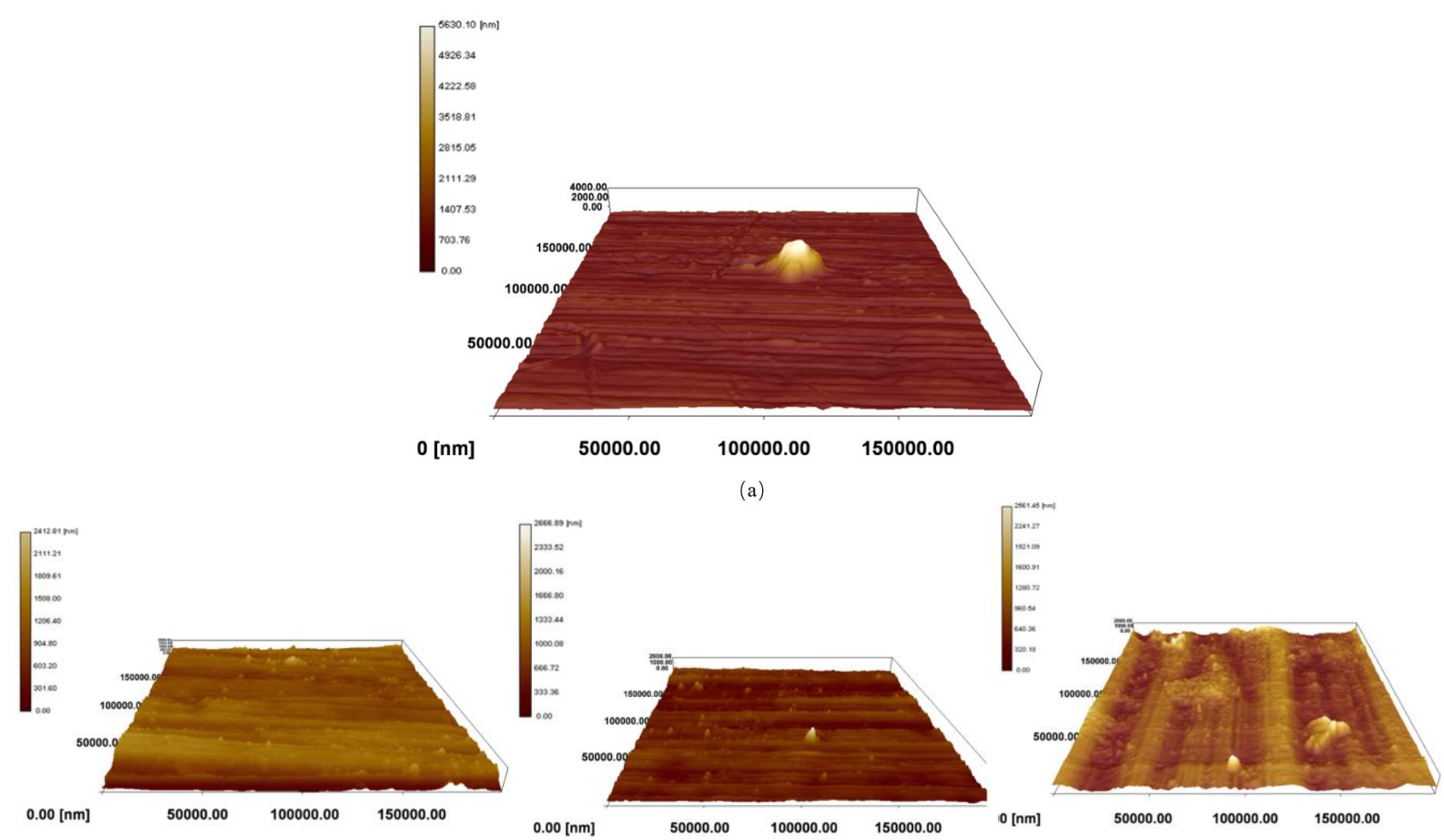

(b)

(c)

(d)

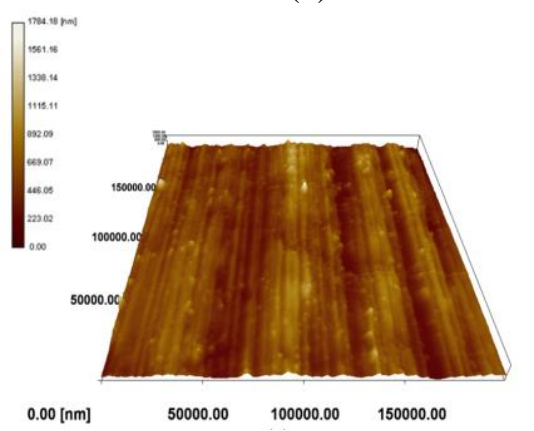

(e) $0.00[\mathrm{~nm}]$

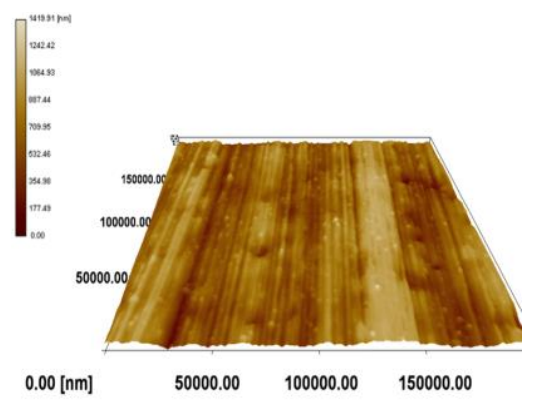

(f)

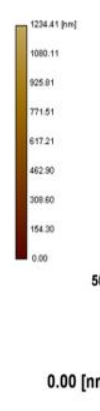

$0.00[\mathrm{~nm}]$

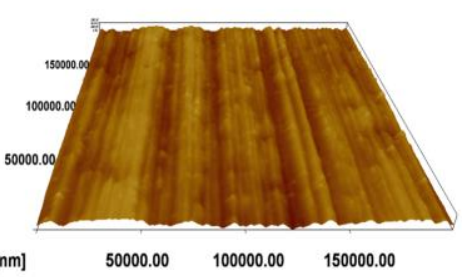

(g)

Fig 7. 3D surface topographies of the surface with different amplitude (a) original specimen, (b) $0.5 \mu \mathrm{m},(\mathrm{c}) 1.00 \mu \mathrm{m}$, (d) $1.5 \mu \mathrm{m}$, (e) $2 \mu \mathrm{m}$,(f) $3.5 \mu \mathrm{m}$, (g) $6 \mu \mathrm{m}$.

Asperity. In this stage, the main deformation could be created by the pre-load from the punch and only a small quantity of asperity's deformation from vibration. So, the height of asperity could still reach about $2666.89 \mathrm{~nm}$ and $2561 \mathrm{~nm}$ under amplitude of 1 and $1.5 \mu \mathrm{m}$ respectively, shown in Fig. 7(c) and (d). Whereas, after increasing amplitude to $2 \mu \mathrm{m}$, which suggested that impact effect would be induced, the further reduction of the height of asperity starts again. As shown in Fig 7(e), the highest asperity is only $1784 \mathrm{~nm}$. When the amplitude increases sequentially to $3.5 \mu \mathrm{m}$ and then $6 \mu \mathrm{m}$, the highest asperity also keeps reducing to $1420 \mathrm{~nm}$ and 1234nm, shown as Fig. 7(f) and (g). it can be explained as more plastic deformation created by the impact effect. The results imply that ultrasonic is useful for improving surface quantity. However, the surface's asperity deformation is basically created by impact effect rather than press machine and acoustic softening is very limited on surface finishing. Additionally, the improvement of surface quantity of deformation of asperity would accumulate gradually and also increasing with a large amplitude of ultrasonic vibration.

\section{Effect on reduction of surface roughness.}

In order to study the reduction of surface roughness by acoustic softening and impact effect, the ratio of reduction of surface roughness $(\Delta \mathrm{Ra})$ was introduced, which can be calculated by following Eq.1:

$$
\Delta R=\frac{R_{0}-R_{a}}{R_{0}} \times 100 \%
$$

(1) the ratio of reduction of surface roughness

Where $R_{0}$ and $R_{a}$ are the surface roughness after the process and before the process.

Three groups of samples are processed by forming process, and averaged values are calculated by five places of surface roughness on every sample about $200 \mu \mathrm{m}^{2}$ measured by AFM as shown in Fig.8. and Fig. 9 shows the relationship between reduction of surface 


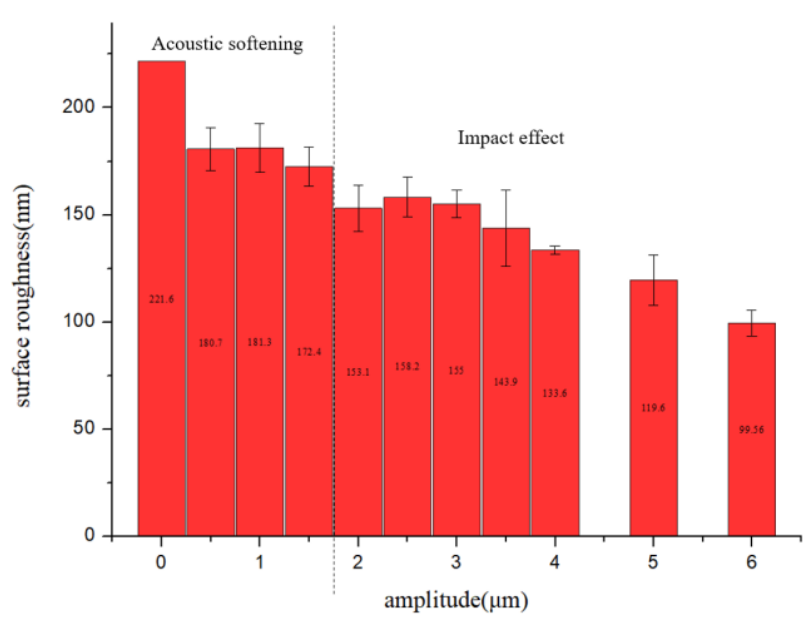

Fig .8 the average surface roughness at different amplitude.

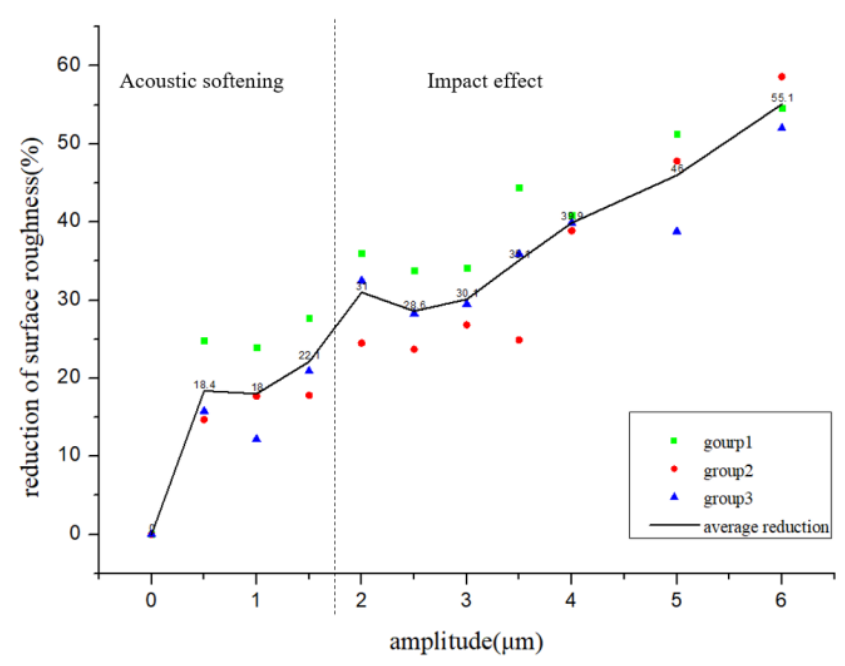

Fig .9 the reduction of surface roughness-amplitude curve.

roughness and different amplitude. Surface roughness is also reduced after ultrasonic vibration started. But only a little change happens on acoustic softening, which highly conforms to a change of surface asperity. The reduction of surface roughness would increase after the impact effect working. Although the reduction is not too much at the initial stage of impact effect working, a more obvious reduction could be obtained. The impact effect also becomes more intense if the amplitude keeps increasing. And finally, about 50\% reduction of surface roughness would be created by the impact effect.

Effect on microstructure after ultrasonic-forging.

To understand how the ultrasonic vibration influences the surface comprehensively, using EBSD to characterize microstructural evolution is necessary. According to the result from EBSD, the difference of acoustic softening and impact effect at the microstructural level could be told more clearly, and it could help us learn the mechanism of ultrasonic vibration on the surface.

Based on the above results, acoustic softening happened at amplitude under $2 \mu \mathrm{m}$ and impact effect start after $2 \mu \mathrm{m}$ and until the end $6 \mu \mathrm{m}$. So the sample with ultrasonic amplitude of 0.5 and $1.5 \mu \mathrm{m}$ are chosen to learn acoustic softening; samples of the ultrasonic amplitude of $2 \mu \mathrm{m}$ and $6 \mu \mathrm{m}$ are chosen to learn the impact effect.

Selected samples were firstly cut from the centerline, and then two places were chosen to be observed. For comparing the difference of ultrasonic vibration influence in surface and entire bulk, one place was close

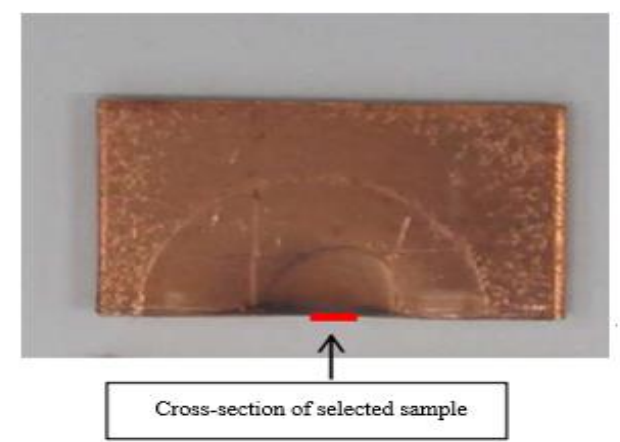

to the surface, another was deeper till to the half of the

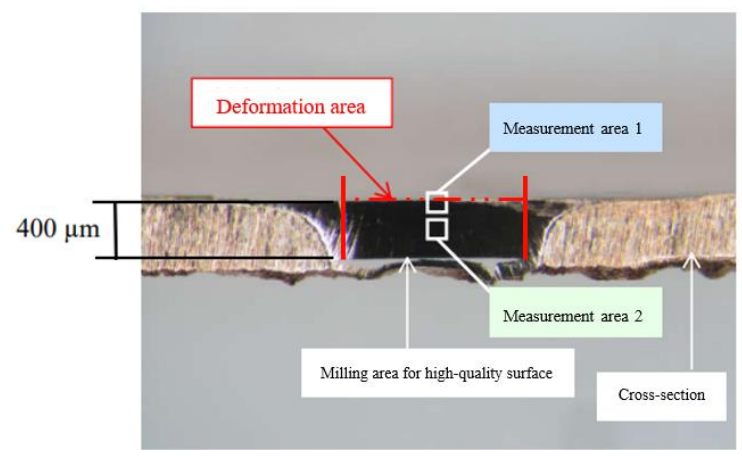

Fig .10 schematic diagram of the EBSD mapping

height of sample, as shown in figure 10.

Comparing with Figure.11(a), which shows the EBSD maps of grain orientation of measurement area 1 and Figure.11(b), which shows measurement area 2, it is easily found that grain size was decreasing gradually with the amplitude increasing and the colorful grain also means more grain rotation in area 1 . But the same trend was not obtained in the center area, indicating that the influence of ultrasonic vibration has a limit. Meanwhile, Figure.11(a) shows that the reduction of average grain size is more evident during amplitude 0.5 to $1.5 \mu \mathrm{m}$, which means acoustic softening is working. It reduced from $13.2 \mu \mathrm{m}$ to $9.3 \mu \mathrm{m}$. Besides, when the amplitude is over to $2 \mu \mathrm{m}$, the trend of reduction of average grain size tends to flatten. Even the maximum amplitude finally reached to $6 \mu \mathrm{m}$; the average grain size is only reduced about $1 \mu \mathrm{m}$ from $9.5 \mu \mathrm{m}$ to $8.6 \mu \mathrm{m}$. Although grain and subgrain could be distinguished when the average grain size decreased by surface deformation, reduction of the average grain size was only a few under low amplitude, and evident shear of grain couldn't be found in area 1 . However, comparing the grain size and form of the grain under amplitude $6 \mu \mathrm{m}$ to the original sample, the change of average grain size is not obvious, but the form of grain has been changed severely. It may be caused by the 

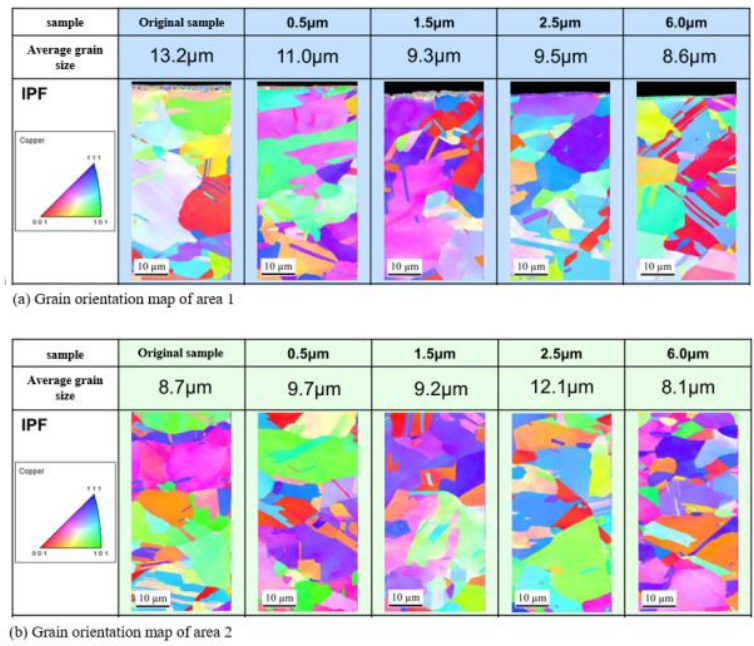

Fig .11 result of the EBSD mapping in two area.

shearing deformation and recrystallization induced by the local heating effect.

For observing the surface more directly, the larger version of the EBSD result on the surface about $5 \mu \mathrm{m}$ depth has been carried out, shown in Figure.12. Before processing, the surface could be classified into three layers by different grain sizes evidently, and the grain size in the upper layer is smaller than the bottom layer, shown in Figure.12 (a). After processing under $1.5 \mu \mathrm{m}$ amplitude, the thickness of layer 2 has become thinner, and most grain in layer 2 has refined to the same size as layer 1, only a few residual grains keep no size change, which suggested the acoustic softening has appeared during vibration, shown as Figure.12 (b). With the further increased ultrasonic to the amplitude of $6 \mu \mathrm{m}$, the results of which is shown in Figure.12(c). It is entirely different from Figure.12 (b). Not only layer 1 has almost disappeared, but also the size of the grain in layer 2 is oppositely elongated. Additionally, the same color of the grain also indicates that the grain keeps in one direction.

During the vibration, from acoustic softening, energy could be offered to the lattice defects, and it drives the defects like the voids or grain boundaries to create more dislocation so that grain obtained refined and more rotation appeared. However, in this process, the energy consumed by the defect closed to the surface and gradually disappeared before it reached the center material, which led to the influence on area 2 is not evident. With the motion of dislocation was enhanced by acoustic softening, the capacity of plastic deformation has also improved, which results in the $50 \mathrm{~N}$ pre-load reading on the date recorder also reduced a little to about $40 \mathrm{~N}$. The same phenomenon was also presented by (Hu and Yang 2017) ${ }^{[7]}$. Although, it still hard to determine if this reduction of stress in micro-forging was induced by acoustic softening or not. As for the impact effect, due to the amplitude increases, the influence of acoustic softening became less. Thousands of times hammering in short period make work hardening happen in the surface microstructure. So, as shown in Figure.12 (c), the grain has been elonged, and no grain refinement occurred. On the other hand, when the impact effect becomes more dominating, the increase of surface temperature could be observed. In the future, what would be created by the heating effect under larger amplitude is worth to be considered.

\section{Conclusion}

In order to study the deformation behavior on the surface of the material, a novel micro-forging test was used, and copper chips were applied to surface finishing by ultrasonic vibration at different amplitudes. Based on the results, it could be concluded as follows:

1. high rigidity of dynamic load cell and oscilloscope could be used to distinguish whether punch detached with surface of specimen in micro-forging. If the waveform on an oscilloscope is displayed as sine, detachment of punch and surface happens, and acoustic softening dominates. Otherwise, the impact effect would be induced. And the distortion of the waveform is more severe, the impact effect is more intense.

2. If there is no detachment of the sample's surface and punch, The deformation is mainly created by acoustic softening, and it is constrained. If detachment of sample's surface and punch happens, deformation of asperity on the surface would increase to a certain degree, which could be attributed to impact effect.

3. Performance of Ultrasonic vibration on surface roughness reducing is highly in line with the
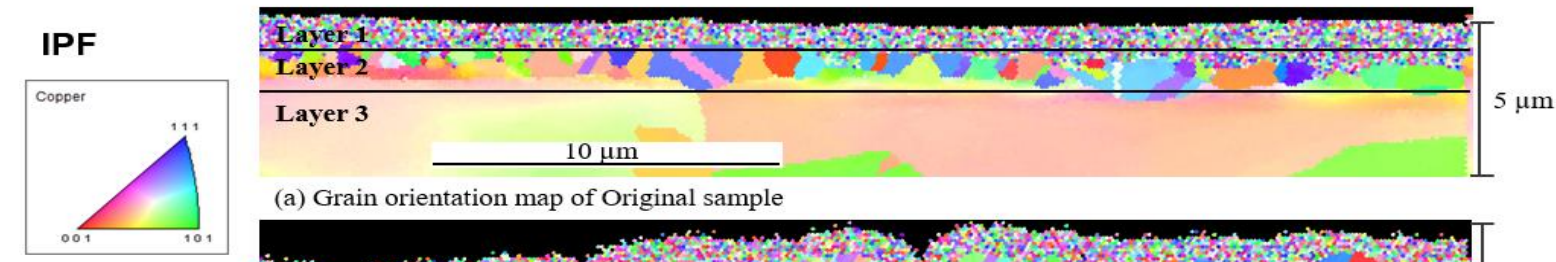

(a) Grain orientation map of Original sample

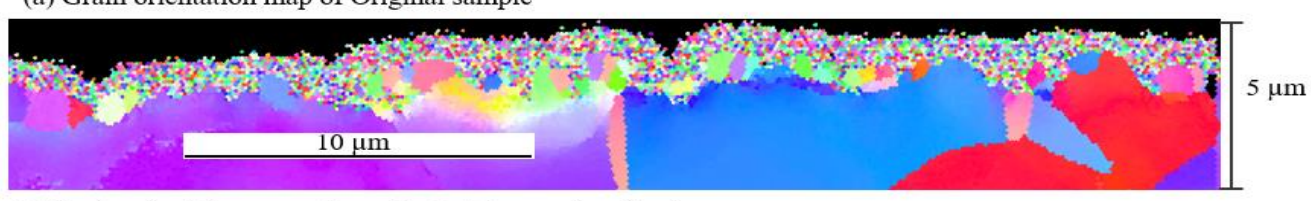

(b) Grain orientation map of amplitude $1.5 \mu \mathrm{m}$ micro-forging

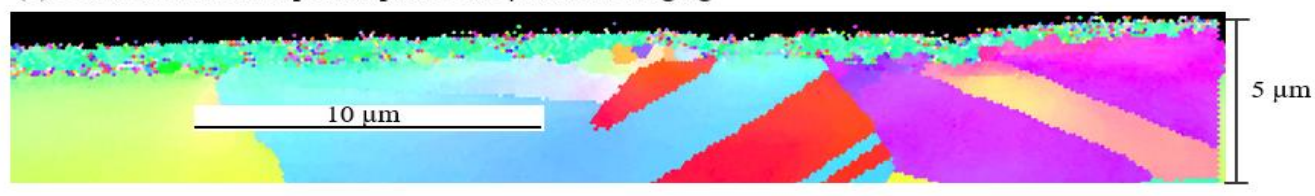

(c) Grain orientation map of amplitude $6 \mu \mathrm{m}$ micro-forging 
change of surface asperity. The result shows impact effect influences the surface roughness more significantly than the acoustic softening. And a reduction of surface roughness created by the impact effect would become more remarkable when impact becomes more dominating with amplitude increasing.

4. The ultrasonic vibration influences the most focus on the surface of material about $5 \mu \mathrm{m}$ depth, and it would decrease progressively from top to $50 \mu \mathrm{m}$ depth.

\section{Acknowledgments}

The authors would like to express their gratitude to Prof. Masahiko JIN (Nippon Institute of Technology) for designing the ultrasonic vibrator. The authors would also like to express their gratitude to Mr.Yohei Suzuki (Komatsuseiki Kosakusho. Co., Ltd.) for designing the compression die.

\section{References}

[1] Yang Bai, Ming Yang, Influence of vibration time and frequency on surface finishing using vibrationassisted micro-forging, Materials Science Forum (2014)687-693.

[2] Yang Bai, Ming Yang, investigation on mechanism of metal foil surface finishing with vibration-assisted micro-forging. Journal of Materials Processing Technology 214.1 (2014): 21-28.

[3] Yang Bai, Ming Yang, optimization of metal foils surface finishing using vibration-assisted micro-forging. [4] Yang Bai, Ming Yang, influence of ultrasonic vibration on metal foils surface finishing with microforging. Procedia Eng. 81(2014)1475-1480.

[5] Jun Hu, Tetsuhide Shimizu, Tomoaki Yoshino, Tomomi Shiratori, Ming Yang. Evolution of acoustic softening effect on ultrasonic-assisted micro/mesocompression behavior and microstructure, Ultrasonic 107(2020)106107

[6] Jun Hu, Tetsuhide Shimizu, Ming Yang, investigation on ultrasonic volume effects: stress superposition, acoustic softening and dynamic impact ultrasonics-sonochemistry.48(2018).240-248.

[7] J. Hu, M. Yang, Impact effect of superimposed ultrasonic vibration on material characteristics in compression tests, Procedia Eng.2017, pp.1063-1068

[8] Vollertsen, F., Biermann, D., Hansen, H.N., Jawahir, I.S., Kuzman, K., 2009. Size effects inmanufacturing of metallic components. CIRP Ann.--Manuf. Technol. 58, 566-587.

[9] Blaha, F., Langenecker, B., 1955. Tensile deformation of zinc crystal under ultrasonic vibration. Naturwissenschaften 42, 556.

[10] Jimma, T., Kasuga, Y., Iwaki, N., Miyazawa, O., Mori, E., Ito, K., Hatano, H., 1998. An application of ultrasonic vibration to the deep drawing process. J. Mater. Process. Technol. 80-81, 406-412.

[11] Weidong Zhai and li Yanle, influence of ultrasonic vibration on the plasticity of metals during compression process. J mater res techno 1. 2020;9(1):433-45
[12] Chunju Wang. Investigations on mechanical properties of copper foil under ultrasonic vibration considering size effect. Procedia Engineering 207 (2017) 1057-106

[13] Bai Yang, Yang Ming, The influence of superimposed ultrasonic vibration on surface asperities deformation, Journal of Materials Processing Technology.

[14] Kirchner, H.O.K., Kromp, W.K., Prinz, F.B., Trimmel, P., 1985. Plastic deformation under simultaneous cyclic and unidirectional loading at low and ultrasonic frequencies. Mater. Sci. Eng. 68, 197206.

[15]Langenecker, B., 1966. Effects of ultrasound on deformation characteristics of metals.IEEE Trans. Sonics Ultrason. 13, 1-8. 